\title{
Event-based Large Scale Surveillance Video Summarization
}

\author{
Xinhui Song, Li Sun, Jie Lei, Guanhong Yuan, Mingli Song \\ College of Computer Science, Zhejiang University, Hangzhou 310027, China
}

\begin{abstract}
Recent advances in sensor manufacture and computer vision technologies have simulated the applications of intelligent transportation systems, while a key yet under-addressed issue in these systems is the semantic summarization of large scale surveillance video. The main difficulty of large scale surveillance video summarization arises from the contradiction between the high-degree spatiotemporal redundancies and the limited storage budget. In this paper, we propose a novel approach of large scale surveillance video summarization on the basis of event detection. In the proposed approach, we firstly obtain the trajectories of vehicles and pedestrians in a tracking-by-detection manner, and then detect the abnormal events using the trajectories. Finally, we design a disjoint max-coverage algorithm to generate a summarized sequence with maximum coverage of interested events and minimum number of frames. Compared with traditional key frame-based approaches, our approach enjoys the following favorable features. First, important information can be efficiently extracted from the redundant contents since the approach is event-centric and those interested events contain almost all the important information. Second, abnormal events are successfully detected by combining the Random Forest classifier and the trajectory features. Third, the abnormal events are designed to display, and hence further reduces the compression ratio. Due to the above features, the proposed approach is suitable for different scenarios, ranges from highway to crowded crossings. Experiments on 12 surveillance sequences validate the effectiveness and efficiency of the proposed approach.
\end{abstract}

Keywords: surveillance video, large scale video summarization, key area

Email address: brooksong@zju.edu.cn (Mingli Song) 
reconstruction

\section{Introduction}

Recent advances in sensor manufacture and computer vision technologies have simulated the applications of intelligent transportation systems with millions of surveillance cameras capturing traffic data every day. Although bringing convenience to human life, the big surveillance data raise the challenge of time and space consuming when retrieving the video. Hence it is necessary to gain certain perspectives of the data without watching the whole video, and video summarization techniques are proposed to handle the problem.

Existing video summarization approaches use key frames to represent the videos as summarization, and most of them $[1,2,3,4,5]$ rely on shot boundary detection. However, in the surveillance data, there are no explicit shot boundaries. Thus it is difficult to decide which frame is more important than others. As a consequence, the summarization results of key framebased methods are not satisfied for surveillance videos. On the other hand, the key frames selected from the original videos may only has a small area with information, while other areas have no valuable information. Hence, the expressivity of key frame-based methods is limited.

In this paper, we propose a novel event-based approach for surveillance video summarization. In contrast with the shot-centric method, our approach is event-centric. On one hand, the proposed approach does not rely on shot boundary detection. On the other hand, since people put much more concerns in the abnormal events than the routines in a surveillance video, our approach works better in extracting the important information from redundant contents and achieves higher summarization ratio than the key frame-based methods.

Specifically, the proposed approach consists of three steps. First, the trajectories of pedestrians and vehicles are obtained by combining the deformable part-based detector and multi-cue data matching in a tracking-bydetection framework. Second, abnormal events in the surveillance videos are detected based on the extracted trajectories. We propose trajectory features, which are discriminative for abnormal event detection when combining with Random Forest classifier. Third, a disjoint max-coverage algorithm is proposed to generate a summarized sequence with maximum coverage of in- 
terested events and minimum number of frames. By this means our approach achieves a very high summarization ratio.

In summary, the main contributions of this work are as follows:

- We propose the event-based video summarization approach, which is more suitable for surveillance video summarization than key framebased approach.

- We combine the Random Forest and the trajectory features to detect abnormal events, and achieve good results.

- We design a disjoint max-coverage algorithm to generate a summarized sequence with maximum coverage of interested events and minimum number of frames

The rest of the paper is organized as follows. Section 2 reviews the related work. Section 3 presents the abnormal events detection and summarization framework. Section 4 demonstrates the experimental results and Section 5 concludes the paper.

\section{Related Work}

\subsection{Abnormal Event Detection}

Existing work on abnormal event detection can be roughly categorized into trajectory analysis and volume matching.

Trajectory analysis-based abnormal event detection approaches track the targets in the videos, stabilize the positions into trajectories, and then recognize whether they are abnormal events. Efros et al. [6] introduce a motion descriptor based on optical flow measurements in a spatiotemporal volume for the tracked human targets, and use an associated similarity measure in a nearest-neighbor framework to recognize the event. Jiang et al. [7] track all moving objects in the video and three different levels of spatiotemporal contexts are considered as features. In each level, frequency-based analysis is performed to automatically discover the rules of the abnormal events. $\mathrm{Wu}$ et al. [8] propose a method for detecting and localizing abnormal events in complicated crowd sequences using a Lagrangian particle dynamics approach, together with chaotic modeling. The method performs well for a range of crowded to sparse scenes. 
Volume matching-based abnormal event detection approaches apply a spatiotemporal volume localization scheme to search for the position of abnormal events. Ke et al. [9] propose to use the volumetric features for video event detection. Spatiotemporal shapes are correlated to video clips, and when combined with flow-based correlation technique, the approach can detect a wide range of actions in video. Rodriguez et al. [10] propose Maximum Average Correlation Height filter to recognize human actions. The filter is capable of capturing intra-class variability by synthesizing a single filter for a given class. Boiman et al. [11] propose ensembles of patches to detect irregularities in videos. They pose the problem of determining the validity of visual data as a process of composing a new observed video segment using chunks of data extracted from previous visual examples. Regions in the observed segments which cannot be composed from the database are regarded as abnormal event. And the problem is formulated as an inference process in a probabilistic graphical model.

\subsection{Video Summarization}

Video summarization techniques aim at summarizing the video with rich imformation into a small number of frames without losing the information.

Most existing video summarization techniques are key frame-based, i.e., several frames from the original videos are extracted to represent the whole video. E.g. Divakaran et al. [2] firstly divide the videos into equilong segments and regard each segment as a shot, and extract the first and the middle frames of the segment as the key frames. Because of its simplicity, this algorithm suffers from redundant contents in the video. Zhang et al. [3] use color histogram as the feature of each frame, and select a key frame if it is significantly differs from the previous chosen one. However, the algorithm cannot guarantee the representativeness of the chosen frames considering the redundancy.

Cernekov et al. [4] proposed to firstly detect shots in the video, and then extract key frames based on the mutual information and the joint entropy. Kelm et al. [5] detect gradual and abrupt cuts to segment the video into shots, and then extract key frames using visual attention features. Liu et al. [1] propose to jointly detect the shot boundaries and extract the key frames in a probabilistic framework, and use Gibbs Sampling to infer the model. Luan et al. [12] select highlight candidate frames from each shot, and then reconstruct the candidate set by nonnegative linear reconstruction. The 
above methods rely on effective shot boundary detection algorithm, which are impractical in surveillance video.

Zhuang et al. [13] propose clustering-based key frame summarization. They first extract color and texture features to represent each frame, and then utilize K-means to obtain several clusters. Finally, if the frame number in a cluster is larger than a threshold, a frame is extracted as the key frame of this cluster. Similarly, Girgensohn et al. [14] also use clustering-based summarization. They require that each key frame should have a neighbor frame that belongs to the same cluster as the key frame, and the time interval between the two frames is less than 9 seconds. Peng et al. [15] propose a graph-based clustering framework for key frame extraction. The similarity between frames is based on maximum matching and optimal matching bipartite graph matching. The clustering-based methods usually ignore the temporal information, which is very important in surveillance video summarization.

Cong et al. [16] provide a content summarization method as a sparsity consistency-based dictionary selection problem, in which a dictionary of key frames is selected such that the original video can be best reconstructed from the dictionary. Sze et al. [17] propose a global statistics-based key frame extraction scheme, where each pixel in the key frame is constructed by considering the probability of occurrence of those pixels at the corresponding pixel position among the frames. Khosla et al. [18] introduce prior information from web images to summarize videos containing a similar set of objects. The above reconstruction-based algorithms suffer from the same problem with clustering-based algorithms, hence the temporal information is lost.

\section{Approach}

Given surveillance videos, we first apply object tracking-by-detection to obtain the trajectories of pedestrians and vehicles, and then use Random Forest to recognize the abnormal events from the trajectories. Finally, a disjoint max-coverage algorithm is proposed to reconstruct the abnormal events. The flowchart of the proposed approach is illustrated in Figure 1.

\subsection{Pedestrian and Vehicle Tracking-by-Detection}

We follow the Tracking-by-Detection framework. The positions of pedestrians and vehicles are first detected and the positions in consecutive frames 


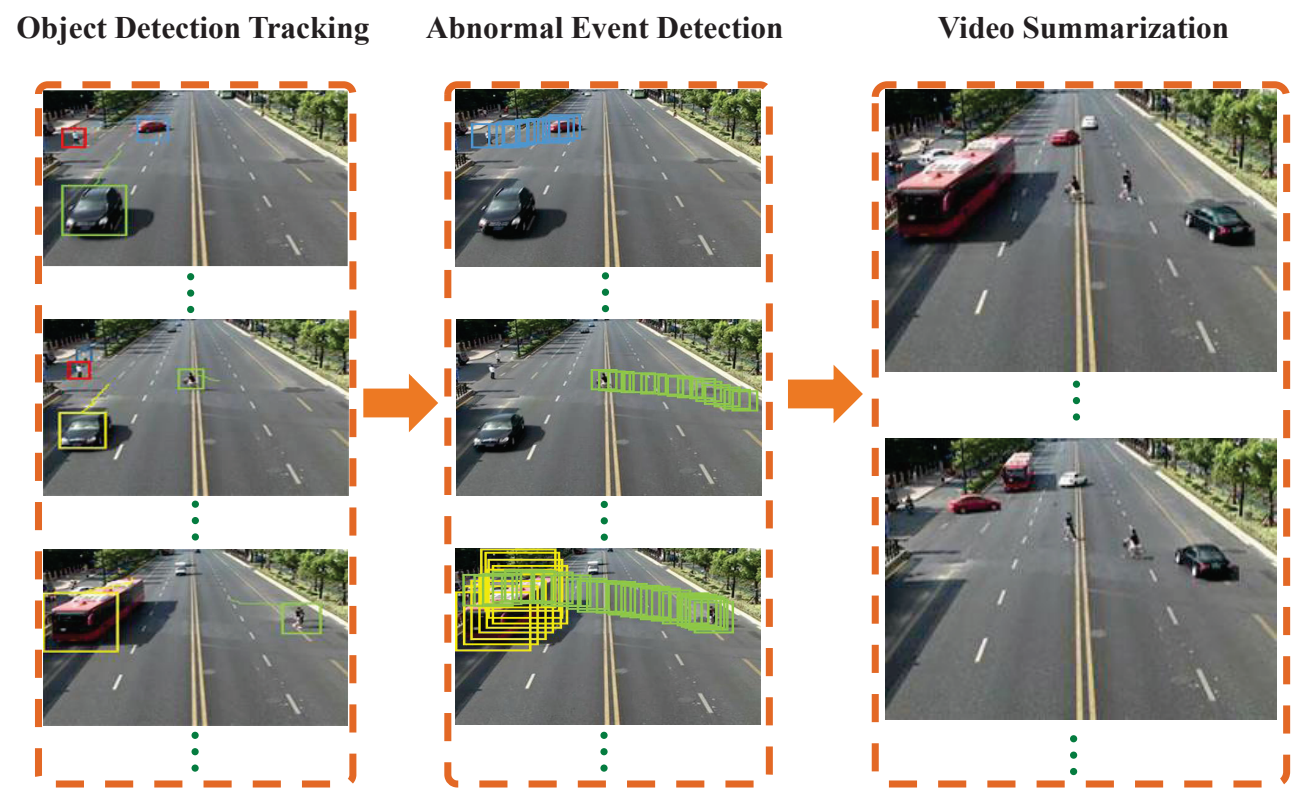

Figure 1: The flowchart of the proposed video summarization approach.

are matched to obtain the trajectories.

The popular deformable part-based model [19] is used for object detection, in which the 31-dimension histogram of oriented gradients (HOG) is used as the feature, and the latent-SVM is used for classification:

$$
f_{\beta}(x)=\max _{z \in Z(x)} \beta \cdot \Phi(x, z)
$$

where $x$ is the HOG feature, $\beta$ is a vector of model parameters, $z$ is a set of latent variables and $\Phi(x, z)$ is the arrangement of the feature and the latent variables.

The score of the model has three parts, i.e., the score of root template, part-based template, and deviation. The first score $S_{\text {core }}$ measures the similarity between the image in a bounding box and the root template of the model, and it could be calculated as the dot product between the feature and the parameter vector of the template. The second score Score $_{p}$ measures the similarity between the subregions of the candidate bounding box and the part-based templates of the model. The third score Score $_{o}$ measures the fitness between the positions of the subregions and the prior positions of the model. The total score is the sum of the three parts. 


$$
\text { Score }=\text { Score }_{r}+\text { Score }_{p}-\text { Score }_{o}
$$

In this paper, we utilize the sliding window scheme to generate bounding boxes in different positions and scales within each testing frame. For each testing bounding box, a cascade algorithm [19] is used to choose the positions of the subregions for part-based classification. If the testing score of the bounding box is larger than a pre-defined threshold, then it is received as a pedestrian or a vehicle according to the classifier.

After object detection, we conduct object tracking algorithm in a greedy data matching framework. Three types of cues are considered to link the the detection responses into trajectories.

Since the same object tends to appear in similar positions in consecutive frames. The position-based matching score is defined as follows:

$$
A_{p}=\exp \left[-\frac{\left(x_{1}-x_{2}\right)^{2}}{\delta_{x}^{2}}\right] \exp \left[-\frac{\left(y_{1}-y_{2}\right)^{2}}{\delta_{y}^{2}}\right]
$$

where $A_{p}$ represents the position-based similarity. $x_{1}$ and $x_{2}$ represent the $\mathrm{x}$-axis coordinates of the the objects in consecutive frames. And $y_{1}$ and $y_{2}$ represent the y-axis coordinates. $\delta_{x}$ and $\delta_{y}$ represent the normalization factors of $\mathrm{x}$-axis and $\mathrm{y}$-axis.

We assume that the size of the same object should be similar in consecutive frames, and obtain the area-based matching score:

$$
A_{s}=\exp \left[-\frac{\left(s_{1}-s_{2}\right)^{2}}{\delta_{s}^{2}}\right]
$$

where $A_{s}$ represents the area-based similarity. $s_{1}$ and $s_{2}$ are the areas of the object detection regions. $\delta_{s}$ is the normalization factor of area.

We also assume the appearance of the same object should be similar in consecutive frames, which leads to the appearance-based matching score:

$$
A_{a}=1-B C\left(D_{1}, D_{2}\right),
$$

where $A_{a}$ represents the appearance-based similarity. $D_{1}$ and $D_{2}$ are the color histograms of two object detection regions and

$$
B C\left(D_{1}, D_{2}\right)=\sum_{l=1}^{Q} \sqrt{D_{1, l}, D_{2, l}}
$$


is the Bhattacharyya coefficient between two histograms [20], where $Q$ is the length of the histogram.

According to the above three factors, the similarity of two object detection regions is decided by the position, area and color histogram. The final matching score of two image regions is defined as the product of the three matching scores:

$$
A=A_{p} A_{s} A_{a}
$$

After getting the similarity between two image regions, the Hungary algorithm [21] is used to find the best match between trajectories and image regions and if the matched score is larger than the threshold $T h$, then the matching is successful. The algorithm initializes a new trajectory if a target is detected and matched successfully in five consecutive frames and terminates an existing trajectory if no objects are found or matched for the trajectory in five consecutive frames.

\subsection{Abnormal Events Detection}

After object tracking, each event is represented as a trajectory. To further detect abnormal event from trajectories, we use the following trajectory features to express a trajectory:

$$
\left[v, d_{x}, d_{y}, x_{s}, y_{s}, x_{t}, y_{t}, x_{m}, y_{m}\right]
$$

where $v$ represents the speed of the object during its moving. $\left(d_{x}, d_{y}\right)$ is the direction of the trajectory. $\left(x_{s}, y_{s}\right),\left(x_{t}, y_{t}\right)$ and $\left(x_{m}, y_{m}\right)$ represent the start, the termination and the intermediate positions of the trajectory, respectively.

The proposed trajectory features are used for abnormal event detection because of its discrimination. E.g., in the surveillance video, if a vehicle changes the lane, the direction of its trajectory changes, and then $\left(x_{s}, y_{s}\right)$, $\left(x_{m}, y_{m}\right)$ and $\left(x_{t}, y_{t}\right)$ cannot be in a line. If the vehicle is over speed, then $v$ will be larger than a threshold. If a vehicle or a pedestrian crosses the street, then the direction of the trajectory is perpendicular to the street, and hence $\left(d_{x}, d_{y}\right)$ is different from most of the trajectories.

To detect the abnormal events from numerous of trajectories, we use the Random Forest [22] to automatically classify the trajectories. Random Forest is natural multiclass classifier, and is suitable for detecting various types of abnormal events. 
Random Forest consists of multiple decision tree classifiers. Each internal node of Random Forest is a binary split function with a partition criterion. Each leaf node of Random Forest is a voter with voters for the class into which the most samples fall.

In the training stage of Random Forest, bagging is conducted to avoid learning overfitting. An individual training set for each tree is generated from the original training set using bootstrap aggregation. For each internal node in the tree, the feature space is binary partitioned using a split function, which leads to the following separate hyperplane:

$$
\mathcal{F}(W, x)=b
$$

where $\mathcal{F}$ is a judging function. $W$ and $b$ are the splitting parameters. The sets of splitting parameters and splitting thresholds are randomly sampled, and the optimal parameter is chosen based on a pre-defined splitting quality measure. In this paper, we use the Gini index [22]:

$$
G(R)=\sum_{k=1}^{K} p_{k}\left(1-p_{k}\right)
$$

where $R$ denotes an internal node, and $p_{k}$ is the probability distribution of the $\mathrm{k}$-th classes in $R$. When used for qualifying splitting, the following function should be maximized:

$$
\triangle G(R, W, b)=G(R)-\frac{\left|R_{l}\right|}{|R|} G\left(R_{l}\right)-\frac{\left|R_{r}\right|}{|R|} G\left(R_{r}\right)
$$

where $R_{l}$ and $R_{r}$ are the left and right child of $R$, respectively.

In the testing stage of Random Forest, a decision tree classifies a test sample by recursively branching binary splits until a leaf node is reached, and the probabilistic output given by the Random Forest is the average categorical posteriors of all the trees.

Specifically, given a testing trajectory $x$, the learned Random Forest predicts whether it is a abnormal event $(y=1)$ or not $(y=0)$ as follows:

$$
p(y=1)=\frac{1}{T} \sum_{t=1}^{T} p_{t}(y)
$$

where $p_{t}(y)$ is the probability given by the t-th tree, which is estimated by calculating the ratio of votes that the abnormal event gets from the leaves in the t-th tree: 


$$
p_{t}(y)=\frac{a_{t}(x)}{a_{t}(x)+n_{t}(x)}
$$

where $a_{t}(x)$ is the number of abnormal events in the leaf of the t-th tree that $\mathrm{x}$ fallen into, and $n_{t}(x)$ is the number of normal events in the leaf of the t-th tree that $\mathrm{x}$ fallen into.

\subsection{Abnormal Events Video Summarization}

After object tracking and abnormal event detection, the testing video now is represented as a set of abnormal events, which is denoted as $E$ :

$$
E=\left\{e_{i} ; i=1 . . N\right\}
$$

where each event $e_{i}$ is represented as a sequence of key areas:

$$
e_{i}=\left\{e_{i, k}, k=1 \ldots L\right\}
$$

where $e_{i, k}$ is the k-th key area of the event. We sample L positions uniformly along the trajectory and for each position the detected bounding box is used as the key area. To best show the abnormal events in limited number of frames, we have three requirements: 1) the least number of frame is used to summarize the video, 2) the showed key areas is disjoint so that the events are clearly displayed and 3) the time order of the key area in a event is preserved. Given the above requirements, we have the following objective function

$$
\begin{array}{cc}
\min _{w_{i, k} \in\{1 \ldots T\}} T \\
\text { s.t. } \forall 1<=t<=T, w_{i_{1}, k_{1}}=t, w_{i_{2}, k_{2}}=t, & \frac{e_{i_{1}, k_{1}} \cap e_{i_{2}, k_{2}}}{e_{i_{1}, k_{1}} \cup e_{i_{2}, k_{2}}}<\eta \\
\text { s.t. } \quad \forall i, k_{1}<k_{2}, & w_{i, k_{1}}<w_{i, k_{2}}
\end{array}
$$

where $w_{i, k}$ indicates the appeared frame index of $e_{i, k}$, and $T$ is the frame number of the summarized video. The first line of (16) means we attempt to use the least number of frame to summarize the video by optimizing the display of key areas. The second line of (16) means that if two key areas appeared in the same frame, they should be disjoint. $\eta$ is a pre-defined ratio. When the ratio of interaction by union of the two areas under eta, they are 


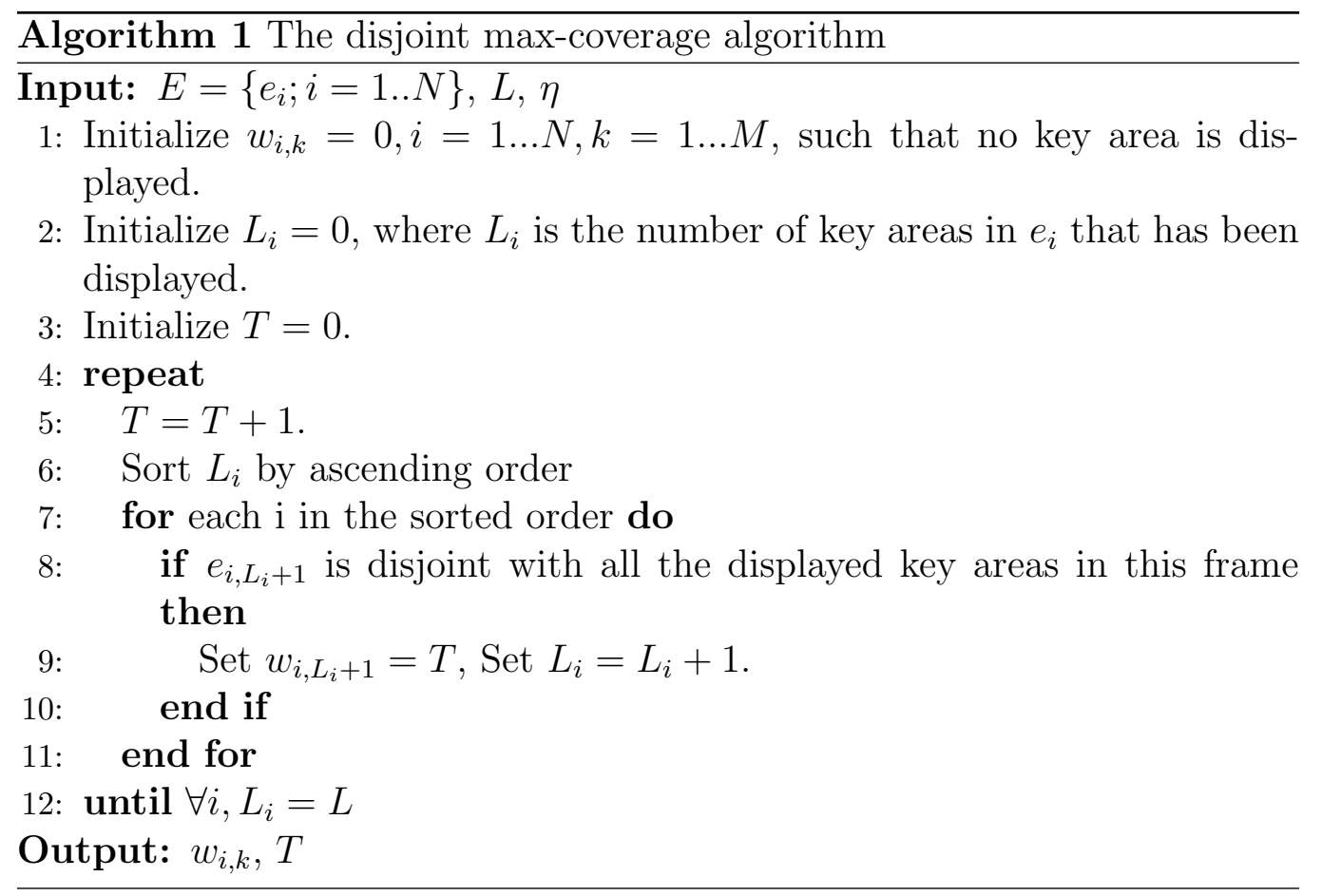

regarded as disjoint. The third line of (16) means that for the same event, the time order of the key area is preserved after summarization.

Since (16) is a multi-variable combination problem, the exact solution is difficult to obtain, and we propose a disjoint max-coverage to get the approximate solution, which is detailed in Algorithm 1.

In particular, the algorithm displays as many key areas as possible for each frame. Because there exist conflicts between key areas, we must define the priorities of the key areas and display them in order of their priorities. In the Algorithm 1, we define $L_{i}$ as the number of key areas in $e_{i}$ that has been displayed. $L_{i}$ is initialized to be zero, since at the beginning of the algorithm, no key areas are displayed. Then for each summarized frame, we sort $L_{i}$ in ascending order, and hence, the event with less displayed key areas is given a higher priority. Considering that if a key area is always conflicting with others, the event is blocked until other events has been summarized, and it will take more frames to summarize the blocked event. The algorithm preferentially displays such key area to prevent this problem.

We use Figure 2 as an example to illustrate the process of the algorith$\mathrm{m}$. The left column shows four events. Each color indicates an event, the 

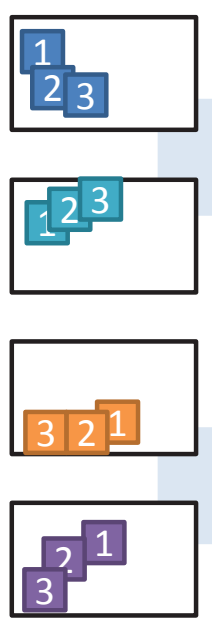
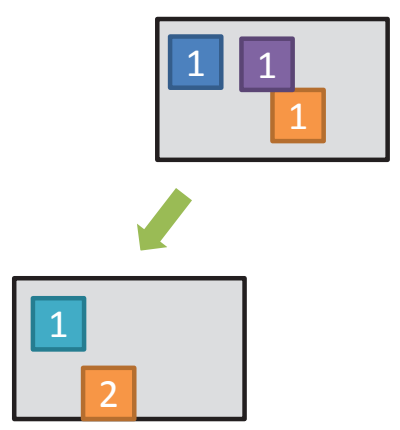

3
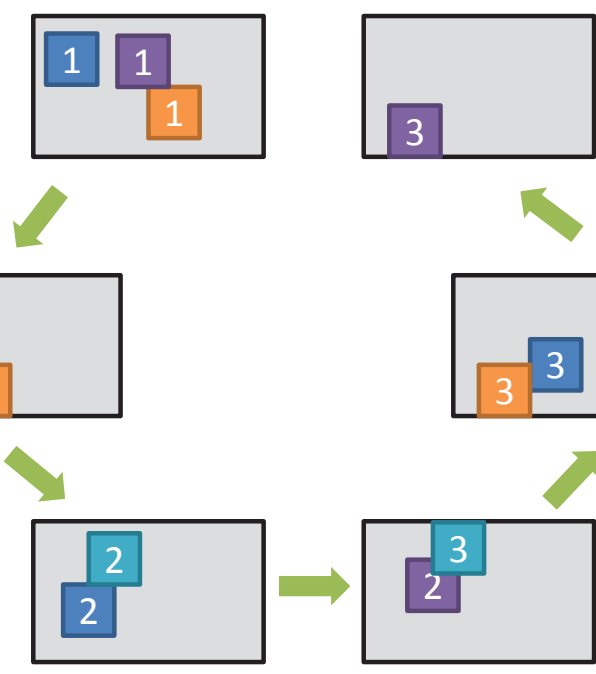

Figure 2: The left column shows four events. Each color indicates an event, the rectangles illustrate the key areas, and the numbers in the rectangles are the time orders of key areas. The right column shows the summarized results.

rectangles illustrates the key areas, and the numbers in the rectangles are the temporal orders of key areas. The right column shows the summarized results. At the first summarized frame, we summarize the first key areas of event 1,3 and 4 into a frame. Since the first key area of event 2 is intersecting with others, it is excluded in the first summarized frame. In the second summarized frame, the first key area of event 2 is chosen since it has the highest priority, and then we display the second key area of event 3 since it is the only one that are not conflicting with event 2 . We then keep summarizing the events until all of the key areas are displayed.

\section{Experiments}

We evaluate the proposed approach on 12 surveillance video sequences: a straight road video (named R1), a highway video (named H1), five crossroad videos (named from $\mathrm{C} 1$ to $\mathrm{C} 5$ ), and five T-junction videos (named from T1 to T5). The lengths of the sequences range from 1 minutes to 60 minutes. For each video sequence, we use the first third part for training, and the rest for testing. None of the trajectories or targets that appears in the testing part is used for training. We compare with the state-of-the-art methods, and 
show that the proposed approach achieves satisfied abnormal event detection and video summarization results.

\subsection{Evaluation of Abnormal Event Detection}

For object detection, we utilize the code of commonly used deformable part-based model [19]. Three types of detectors are trained: pedestrian, vehicle, and riding people. The thresholds of the detectors are set to -0.3 , -0.1 and -0.4 to ensure the recall of each category is larger than 0.9. For object tracking, $\delta_{x}$ and $\delta_{y}$ are set to $20, \delta_{s}$ is set to 6 and $T h$ is set to 10000 through cross-validation.

For abnormal event detection, 100 trees are used to build the Random Forest and 100 hyperplanes are randomly generated for each internal node. Five types of judging functions are considered: 1) the value of a feature, 2) the sum of two features, 3) the difference of two features, 4) the absolute difference of two features and 5) the linear combination of multiple features. The type of the judging function is randomly sampled.

For the training and testing video, we firstly apply object tracking-bydetection to obtain the trajectories and then hand-label whether the trajectory is a abnormal event. 3508 trajectories obtained from the training videos are labeled for training the Random Forest classifier, 6972 trajectories are labeled in the testing videos. About 1/10 trajectories are labeled to be abnormal. By changing the threshold of the Random Forest classifier, we obtain the ROC curve for abnormal event detection. The result is shown in Figure 3.

We also test combining the trajectory features and other state-of-the-art classifiers for abnormal event detection. The results of linear Support Vector Machine (SVM) [23] and AdaBoost [24] are shown in Figure 3. Obviously, the Random Forest achieves the best results among them.

We compare with [16] and [7]. The results are summarized in Table 1 and the results are measured by the area under ROC (namely, AUC). It is easy to find that our AUC outperforms others.

Figure 4 gives some detected samples of our algorithm. The first column shows the lane changing events, the second column shows the over speed events, and the third column shows the jaywalk events. Each picture shows the trajectory of an abnormal event, and the bounding boxes are the detected object positions of the trajectory. As can be seen, our algorithm successfully detect the abnormal events as expected. 


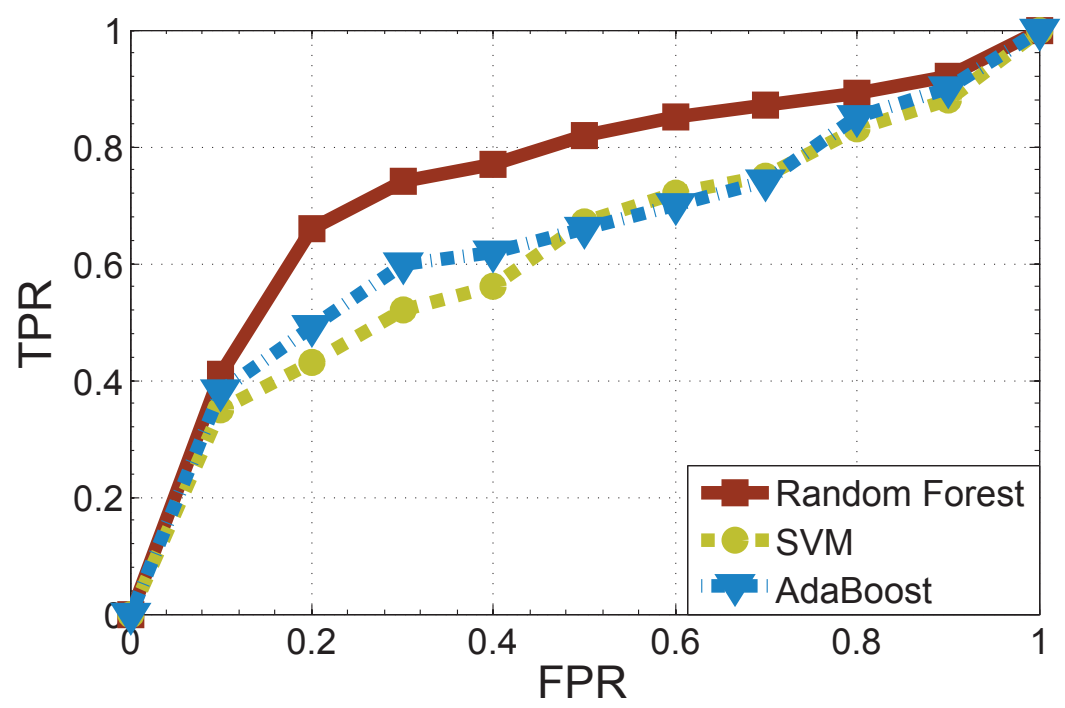

Figure 3: The ROC curves for abnormal event detection by combining the trajectory features and Random Forest, SVM and AdaBoost.

\begin{tabular}{c||c}
\hline Method & AUC \\
\hline \hline Cong et al. [16] & $69.8 \%$ \\
Jiang et al. [7] & $65.3 \%$ \\
ours & $74.3 \%$ \\
\hline
\end{tabular}

Table 1: mAP scores of different weakly supervised segmentation methods on the YTO dataset.

\subsection{Evaluation of Video Summarization}

For video summarization, $L$ is set to 3 and hence the trajectory of each abnormal event has 3 key regions. Meanwhile, $\eta$ is set to 0.2 . We adjust the threshold of Random Forest classifier to ensure that the recall of abnormal event detection is larger than $90 \%$.

In our implementation, to preserve the spatial continuity of the abnormal events, the algorithm does not summarize all the abnormal all at once, but summarizes the abnormal events happened in one minute in each time.

We use compression ratio to measure the performance of the summarization algorithm. The compression ratio is defined as the number of original frames over the number of summarized frames.

Figure 5 shows the compression ratio of different sequences using the 


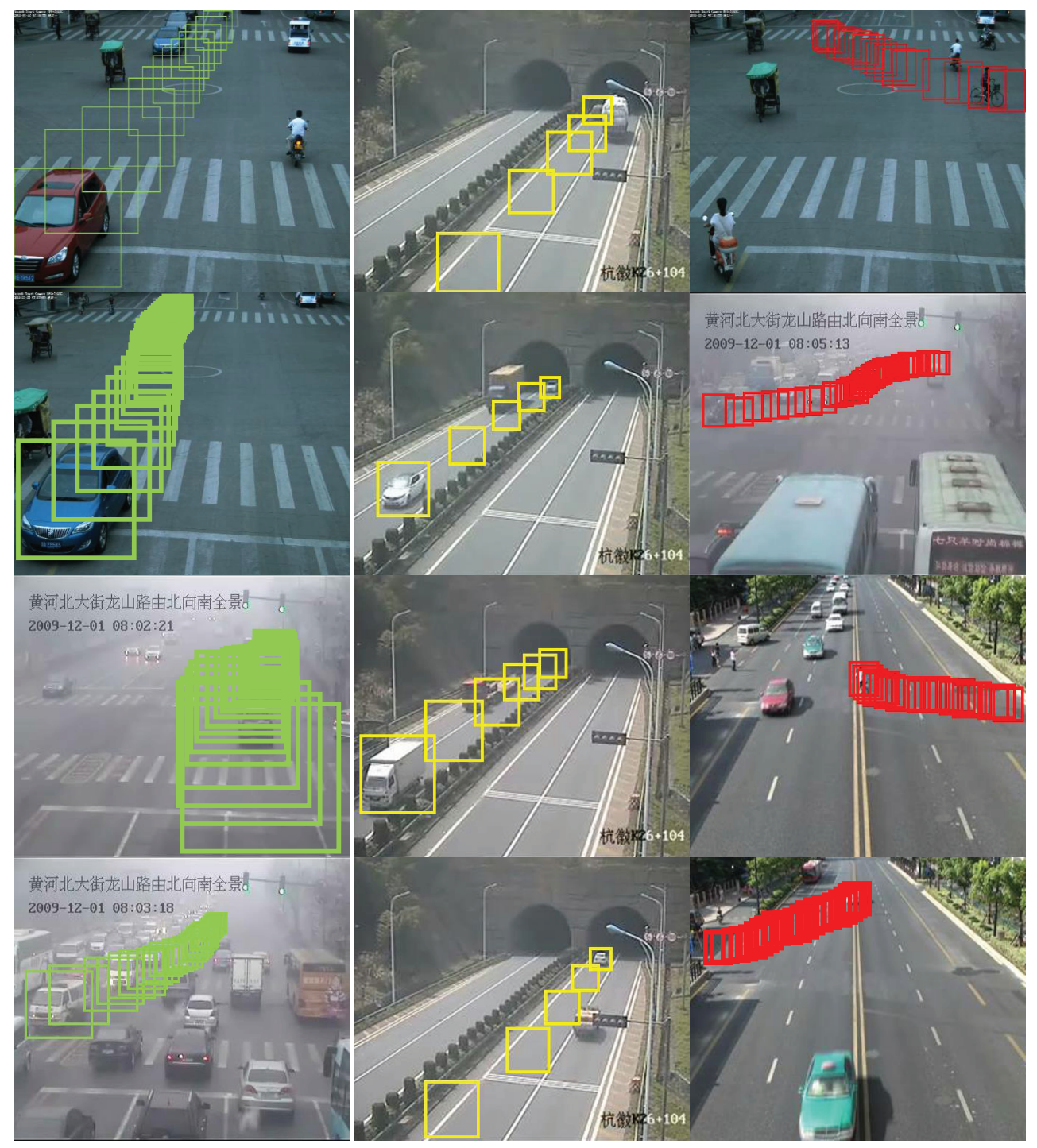

Figure 4: Samples of abnormal events using our algorithm. The first column shows the lane changing events, the second column shows the over speed events, and the third column shows the jaywalk events. Each picture shows the trajectory of an abnormal event, and the bounding boxes are the detected object positions of the trajectory. 


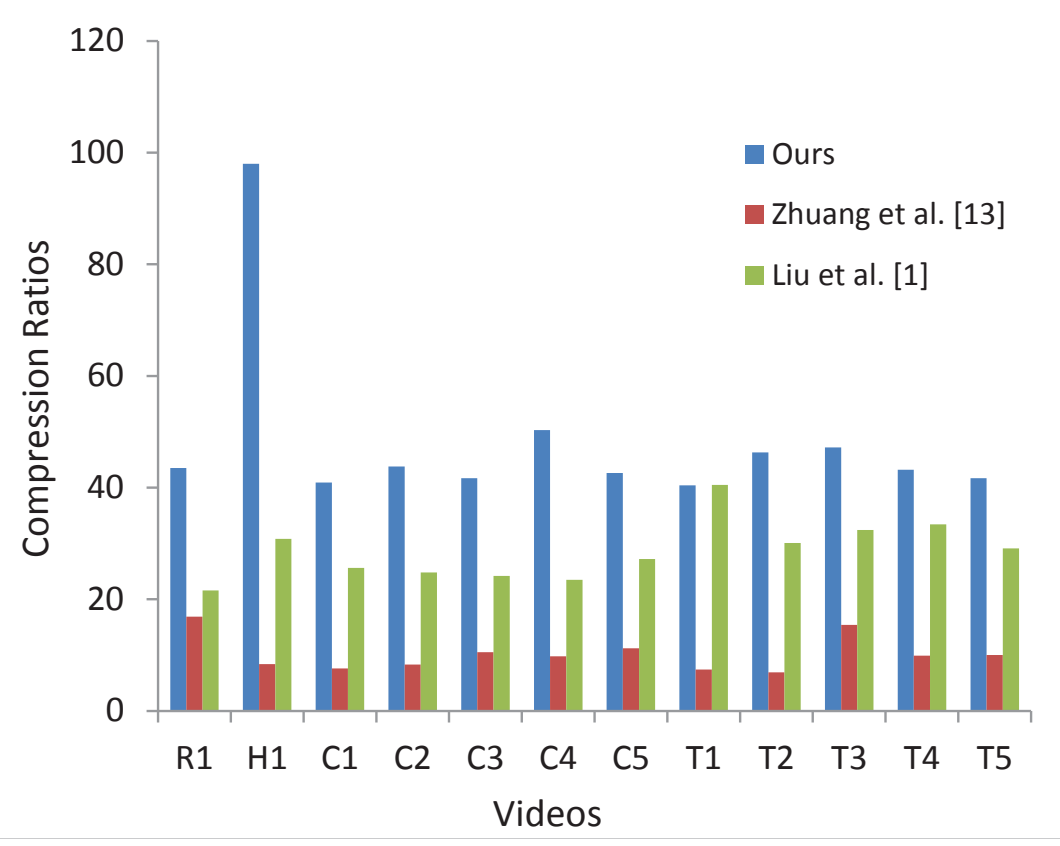

Figure 5: The compression ratio of the proposed approach, [7] and [1].

proposed approach. Our approach achieves very high compression ratios. In the highway sequence, the compression ratio is as high as 98 .

We compare with a clustering-based key frame approach [7] and a shot boundary detection-based key frame approach [1]. From Figure 5, the proposed approach outperforms others. [1] has similar compression ratio with our approach in $\mathrm{T} 1$ and T4. However, its summarized key frames have lost much information.

Figure 6 shows the samples of summarized results of the proposed approach, [7] and [1]. The first line shows the results of the proposed approach, the second shows the results of [7] and the third line shows the results of [1]. As can be seen, our approach uses five frames successfully for eight abnormal events, while [7] and [1] fail to capture enough abnormal events.

We provides more summarized samples in Figure 7.

\section{Conclusion}

This paper focuses on the problem of surveillance video summarization. While traditional video summarization methods rely on key frame extraction, 


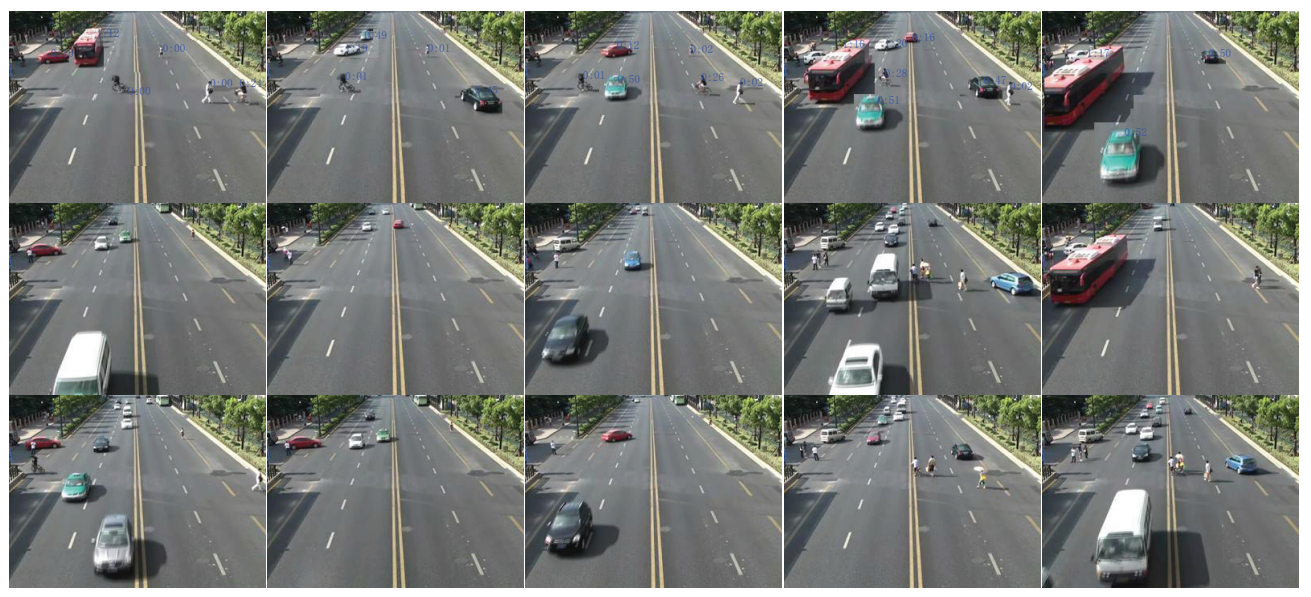

Figure 6: Samples of summarized results of R1. The first line shows the results of the proposed approach, the second shows the results of [7] and the third line shows the results of [1]. As can be seen, our approach uses five frames successfully for eight abnormal events, while [7] and [1] fail to capture enough abnormal events.

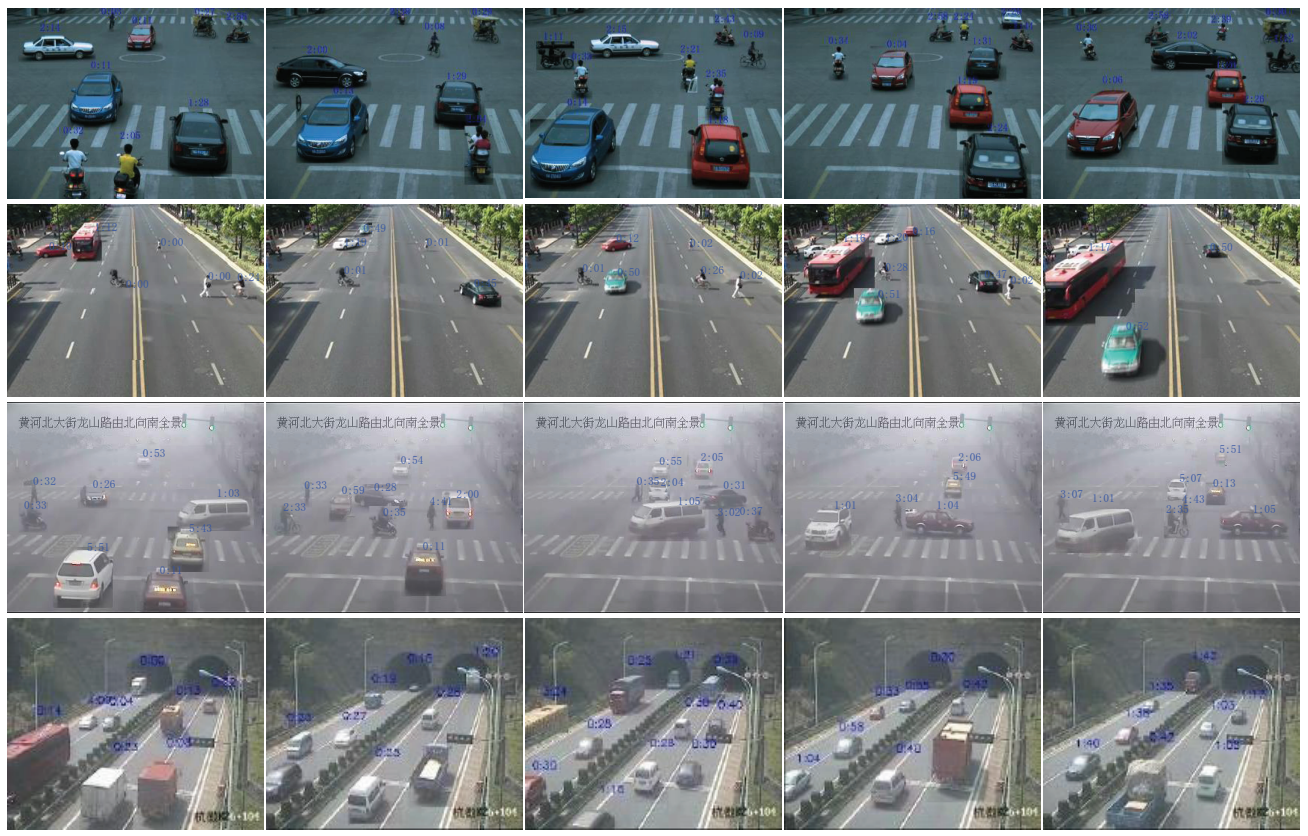

Figure 7: The samples of summarized results of T1, R1, C1, and H1 using the proposed approach. The time stamp shown on the top of each target is the time that it appears in the original video. 
we propose an event-based approach to summarize the surveillance videos. Compared with key frame-based methods, our approach has the following advantages: 1) it has higher summarization ratio, 2) it captures the abnormal events in the surveillance, and hence it preserves the most important semantic information of the videos, and 3) it does not rely on shot boundary detection, which is difficult to conduct in surveillance videos.

To detect the abnormal events in the surveillance video, we firstly apply object tracking-by-detection to obtain the trajectories of pedestrians and vehicles. The abnormal events are then detected by combining trajectory features and Random Forest. To summarize the abnormal events into a limited number of frames, we propose the disjoint max-coverage algorithm for best displaying. The blocked events are given higher priorities to decrease the used summarized frames.

The proposed approach is suitable for different scenarios, ranging from highway to crowded crossings. It is automatic, fast and robust. In the future, we shall combine the key frame-based and event-based summarization approaches to further increase the summarization ratio. We will also try our algorithm in more types of abnormal events or other types of videos.

\section{Acknowledgements}

This work was supported in part by the the Program of International S\&T Cooperation (2013DFG12840), by the National Science Foundation of China (61170142), by the National High Technology Research and Development Program of China (2013AA040601)

\section{References}

[1] X. Liu, M. Song, L. Zhang, S. Wang, J. Bu, C. Chen, D. Tao, Joint shot boundary detection and key frame extraction, in: ICPR, 1-4, 2012.

[2] A. Divakaran, R. Radhkrishnan, A. Peker, Motion activity-based extraction of key-frames from video shots, in: ICIP, 1-4, 2002.

[3] H. Zhang, J. Wu, D. Zhong, S. Smoliar, An integrated system for content-based video retrieval and browsing, Pattern Recognition 30 (1997) 643-658. 
[4] Z. Cernekov, I. Pitas, C. Nikou, Information Theory-Based Shot Cut/Fade Detection and Video Summarization, IEEE Transactions on Circuits and Systems for VIdeo Technology (1) (2006) 82-91.

[5] P. Kelm, S. Schmiedeke, T. Sikora, Feature-based video key frame extraction for low quality video sequences, in: 10th Workshop on WIAMIS, 25-28, 2009.

[6] A. Efros, A. Berg, G. Mori, J. Malik, Recognizing actions at a distance, in: ICCV, 2003.

[7] F. Jiang, J. Yuan, S. Tsaftarisa, A. Katsaggelosa, Anomalous video event detection using spatiotemporal context, Computer Vision and Image Understanding (2011) 323-333.

[8] S. Wu, B. Moore, M. Shah, Chaotic Invariants of Lagrangian Particle Trajectories for Abnomaly Detection in Crowded Scenes, in: CVPR, 2010.

[9] Y. Ke, R. Sukthankar, M. Hebert, Volumetric features for video event detection, International Journal of Computer Vision (2010) 339-362.

[10] M. Rodriguez, J. Ahmed, M. Shah, Action mash: Aspatio-temporal maximum average correlation height filter for action recognition, in: CVPR, 2008.

[11] O. Boiman, M. Irani, Detecting Irregularities in Images and in Video, International Journal of Computer Vision (2007) 1-8.

[12] Q. Luan, M. Song, C. Liao, J. Bu, Z. Liu, M. Sun, Video summarization based on nonegative linear reconstruction, in: ICME, 1-6, 2014.

[13] Y. Zhuang, Y. Rui, T. S. Huang, S. Mehrotra, Adaptive key frame extraction using unsupervised clustering, in: ICIP, vol. 1, 866-870, 1998.

[14] A. Girgensohn, A fast layout algorithm for visual video summaries, in: ICME, 77-80, 2003.

[15] Y. Peng, C. Ngo, Clip-based similarity measure for query-dependent clip retrieval and video summarization, IEEE Transactions on Circuits and Systems for VIdeo Technology 16 (5) (2006) 612-627. 
[16] Y. Cong, J. Yuan, J. Luo, Towards scalable summarization of consumer videos via sparse dictionary selection, IEEE Transactions on Multimedia 14 (1) (2012) 66-75.

[17] K. Sze, K. Lam, G. Qiu, A new key frame representation for video segment retrieval, IEEE Transactions on Circuits and Systems for VIdeo Technology 15 (9) (2005) 1148-1155.

[18] A. Khosla, R. Hamid, C. Lin, N. Sundaresan, Large-scale video summarization using web-image priors, in: CVPR, 2698-2705, 2013.

[19] P. Felzenszwalb, D. McAllester, D. Ramanan, A discriminatively trained, multiscale, deformable part model, in: CVPR, 1-8, 2008.

[20] D. Comaniciu, V. Ramesh, P. Meer, Kernel-based object tracking, Pattern Analysis and Machine Intelligence, IEEE Transactions on 25 (5) (2003) 564-577.

[21] B. Wu, R. Nevatia, Detection and tracking of multiple, partially occluded humans by Bayesian combination of edgelet based part detectors, International Journal of Computer Vision (2007) 247-266.

[22] L. Breiman, Random Forests, in: Machine Learning, 5-32, 2001.

[23] C. Chang, C. Lin, LibSVM: a library for support vector machines, ACM Transactions on Intelligent Systems and Technology (2011) 1-27.

[24] P. Viola, M. Jones, Rapid object detection using a boosted cascade of simple features, in: CVPR, 2001. 
Xinhui Song received the B.E. degree in Software Engineering from Tianjin University, in 2014, and is pursuing the M.S. degree in Microsoft Visual Perception Laboratory, Zhejiang University. Her research interests include deep learning and video understanding.

Li Sun is currently a PhD student in the college of Computer Science, Zhejiang University, Zhejiang, China. His research interests include computer vision and machine learning.

Jie Lei is currently a PhD student in the College of Computer Science, Zhejiang University, Zhejiang, China. His research interests include computer vision, robotic vision and machine learning.

Dapeng Tao received a B.E degree from Northwestern Polytechnical University and a Ph.D. degree from South China University of Technology, respectively. He is currently with School of Information Science and Engineering, Yunnan University, Kunming, China, as an engineer. He has authored and co-authored more than 30 scientific articles. He has served more than 10 international journals including IEEE TNNLS, IEEE TMM, IEEE SPL, and PLOS-ONE. Over the past years, his research interests include machine learning, computer vision and cloud computing.

Guanhong Yuan received the B.E. degree in Computer Science from Central South University, in 2012, and M.S. degree in Microsoft Visual Perception Laboratory, Zhejiang University, in 2015. Her research interests is computer vision.

Mingli Song (M'06-SM'13) is a Professor in Microsoft Visual Perception Laboratory, Zhejiang University. He received the $\mathrm{PhD}$ degree in Computer Science from Zhejiang University, China, in 2006. He was awarded Microsoft Research Fellowship in 2004. His research interests include face modeling and facial expression analysis. 
"Photo of Xinhui

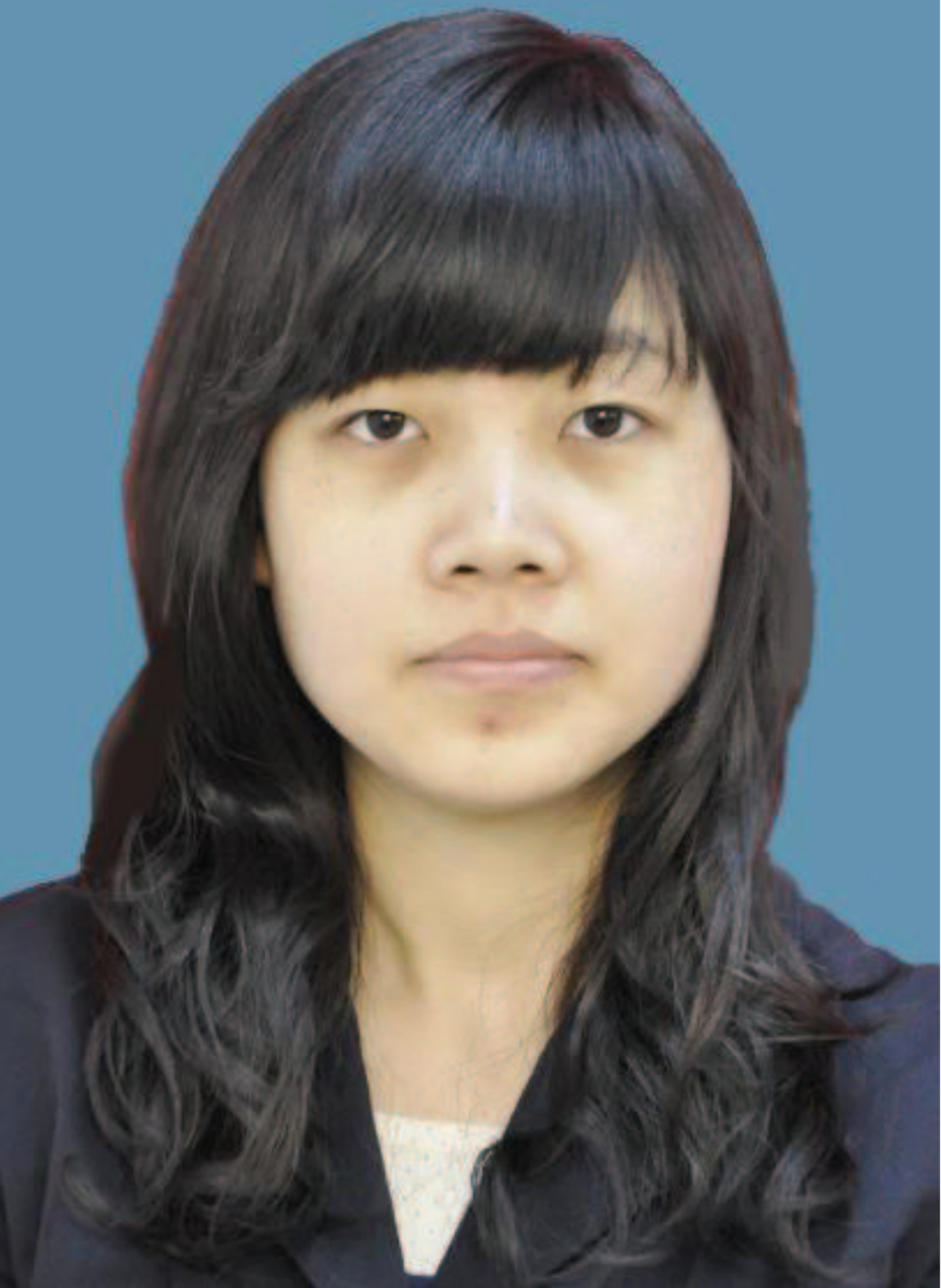




\section{*Photo ofSong}

\section{.}

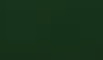

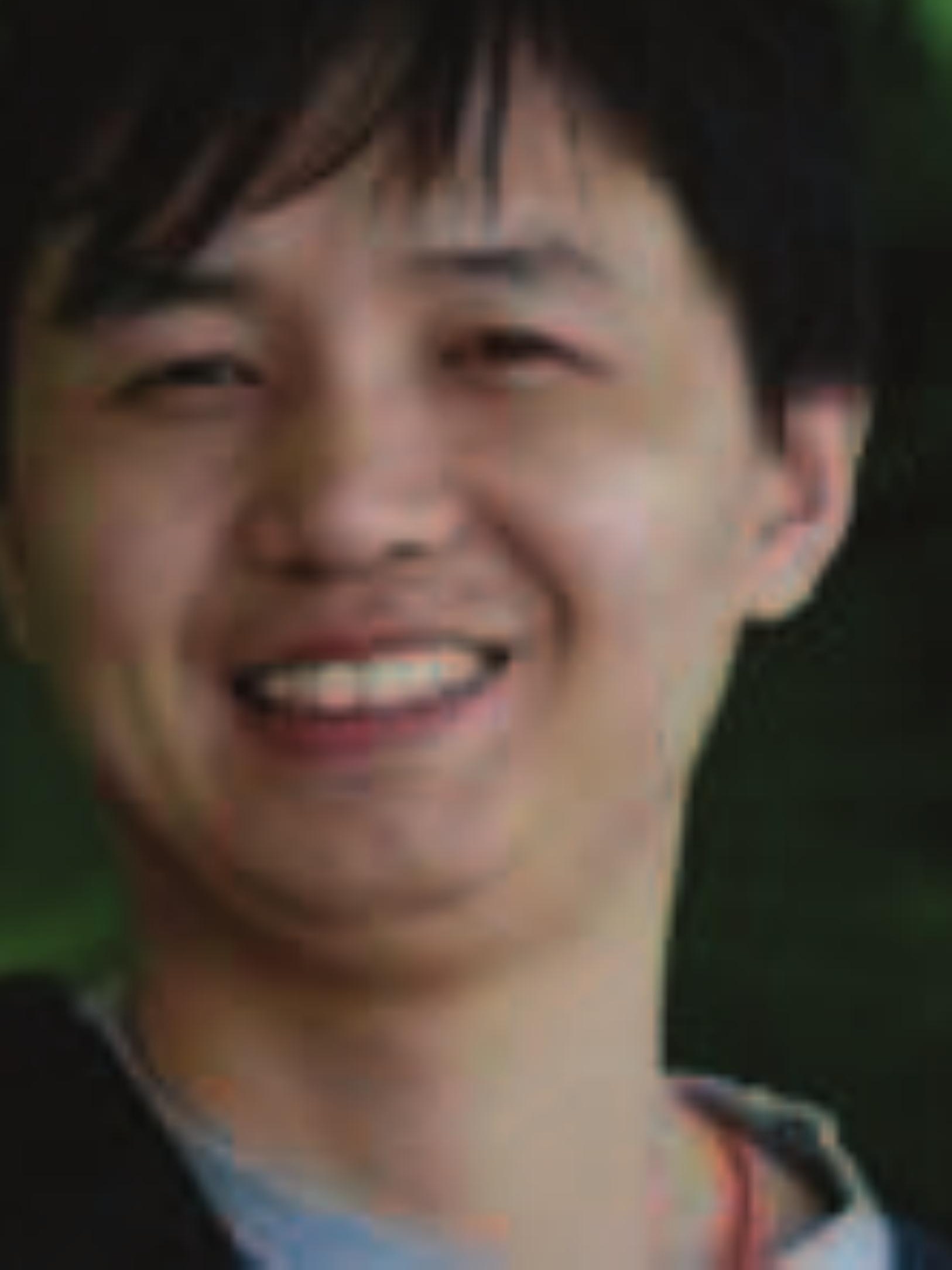

(


${ }^{*}$ Photo of Sun

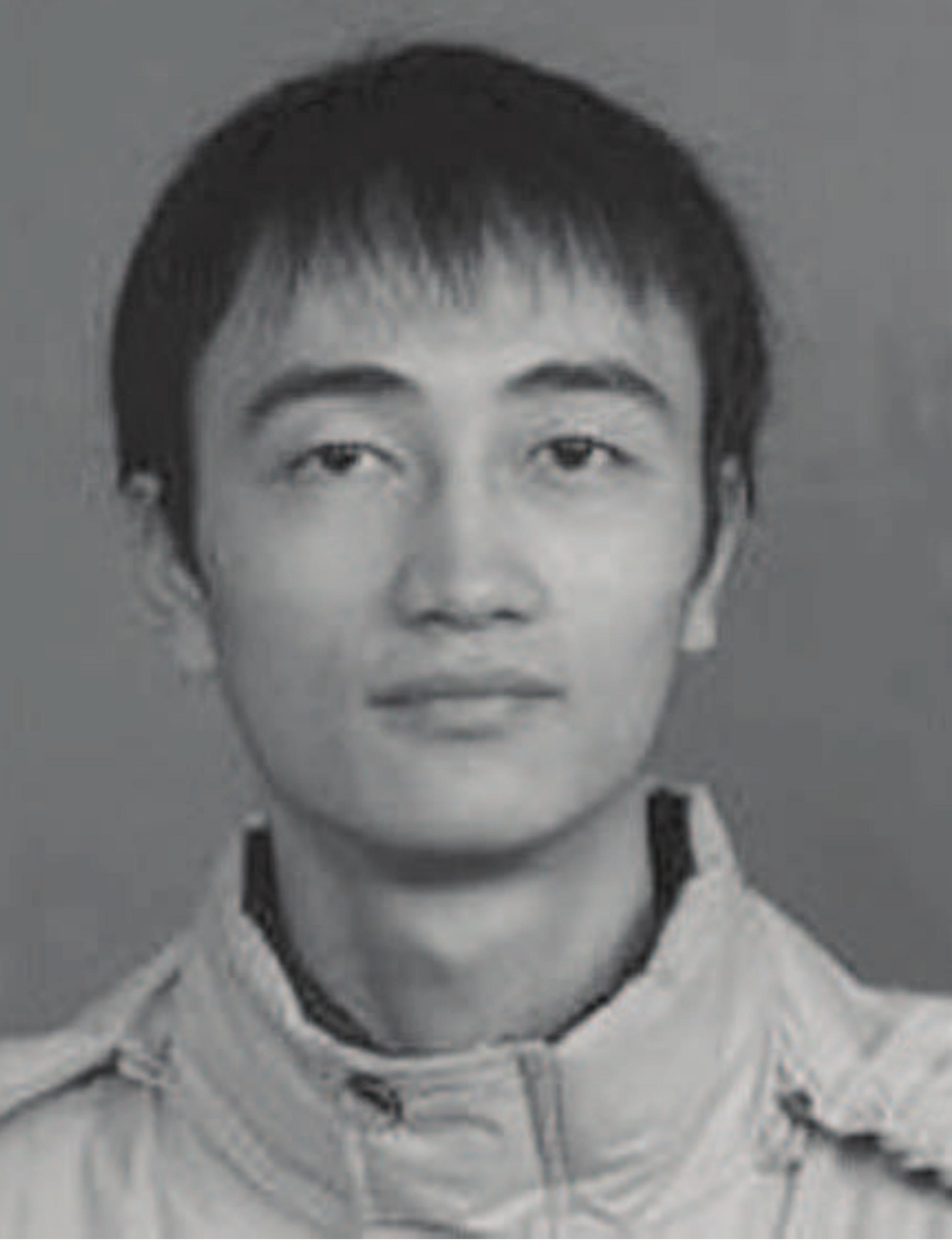


"Photo of Yuan

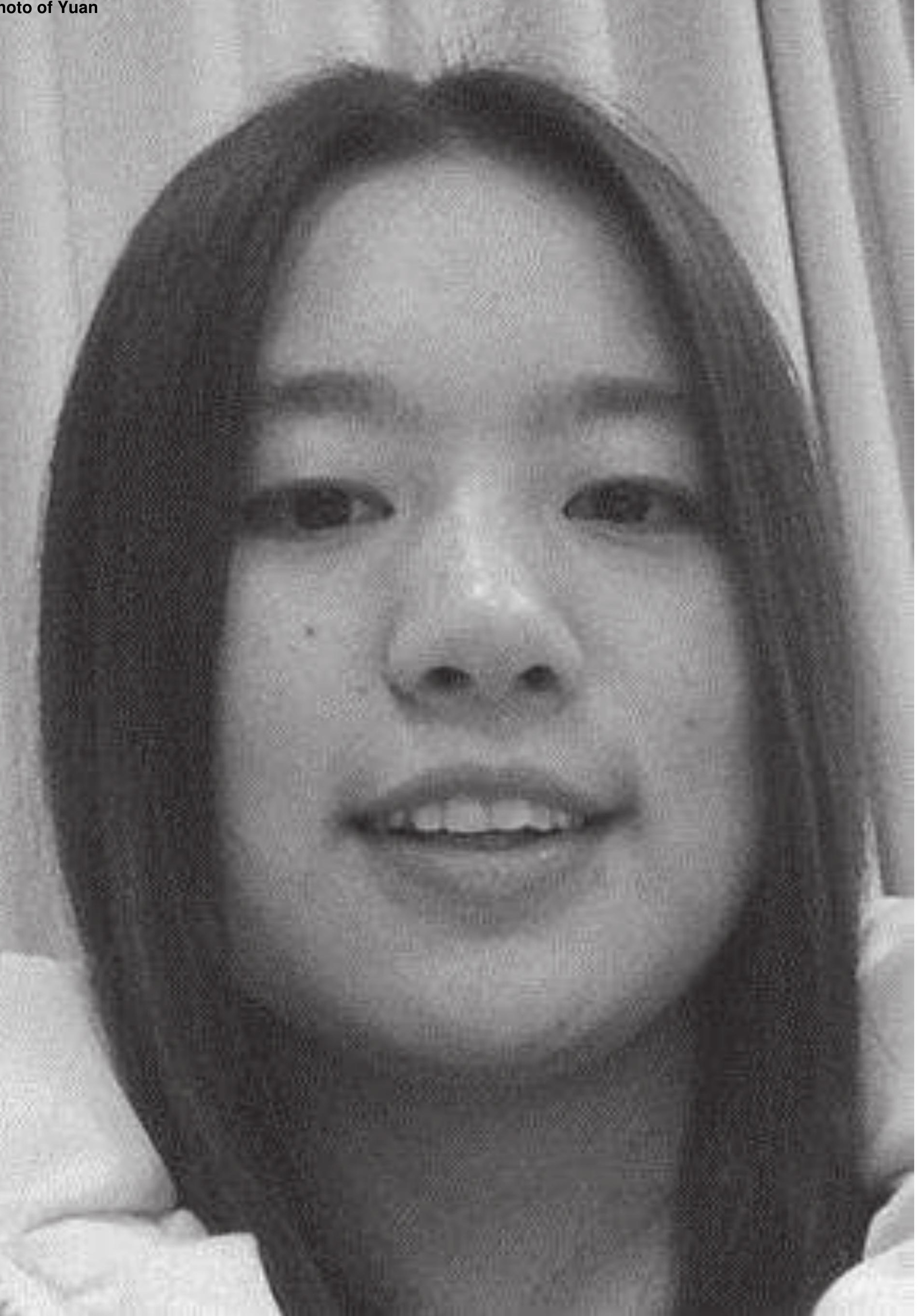




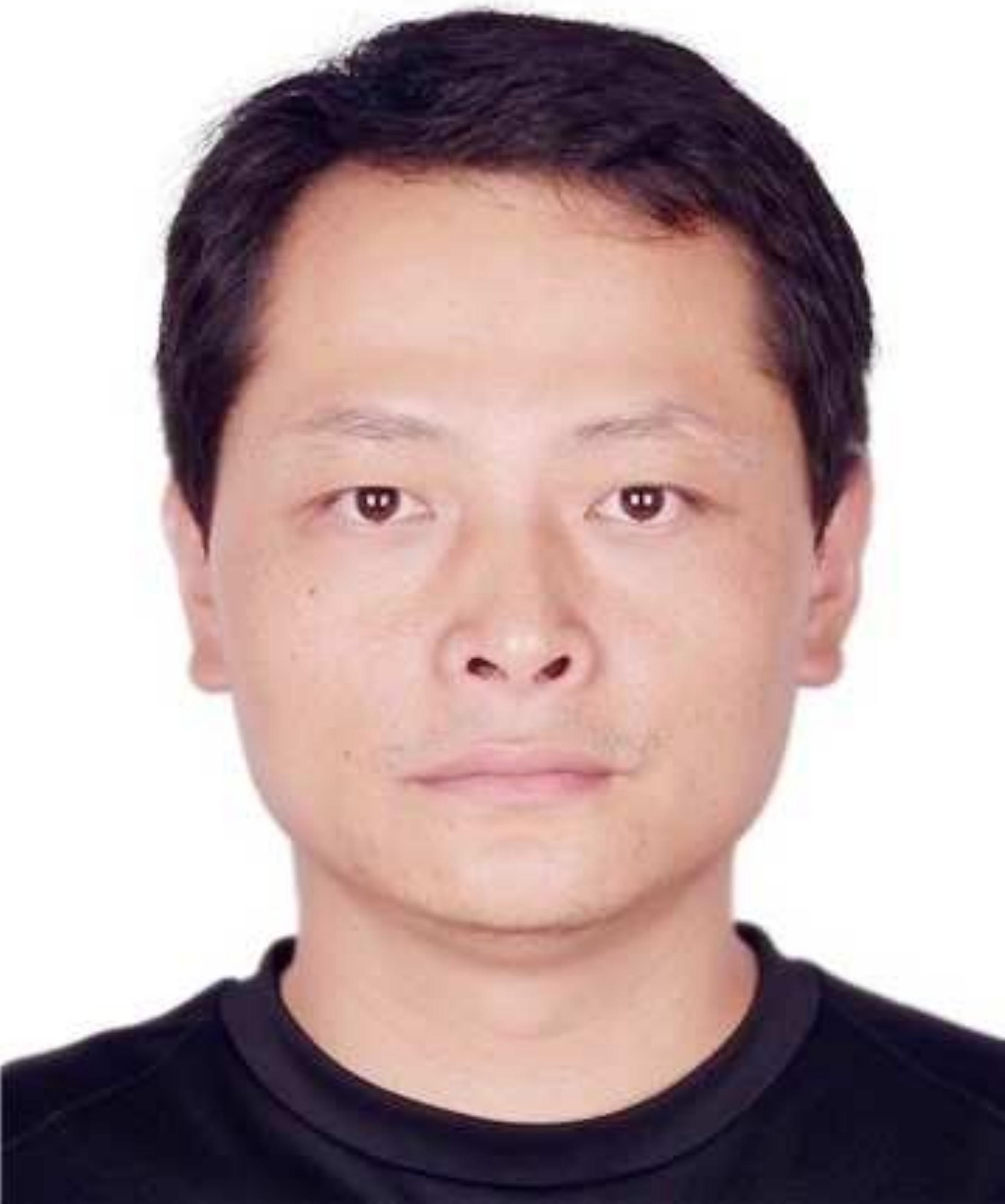

${ }^{*}$ Photo of DapengTao
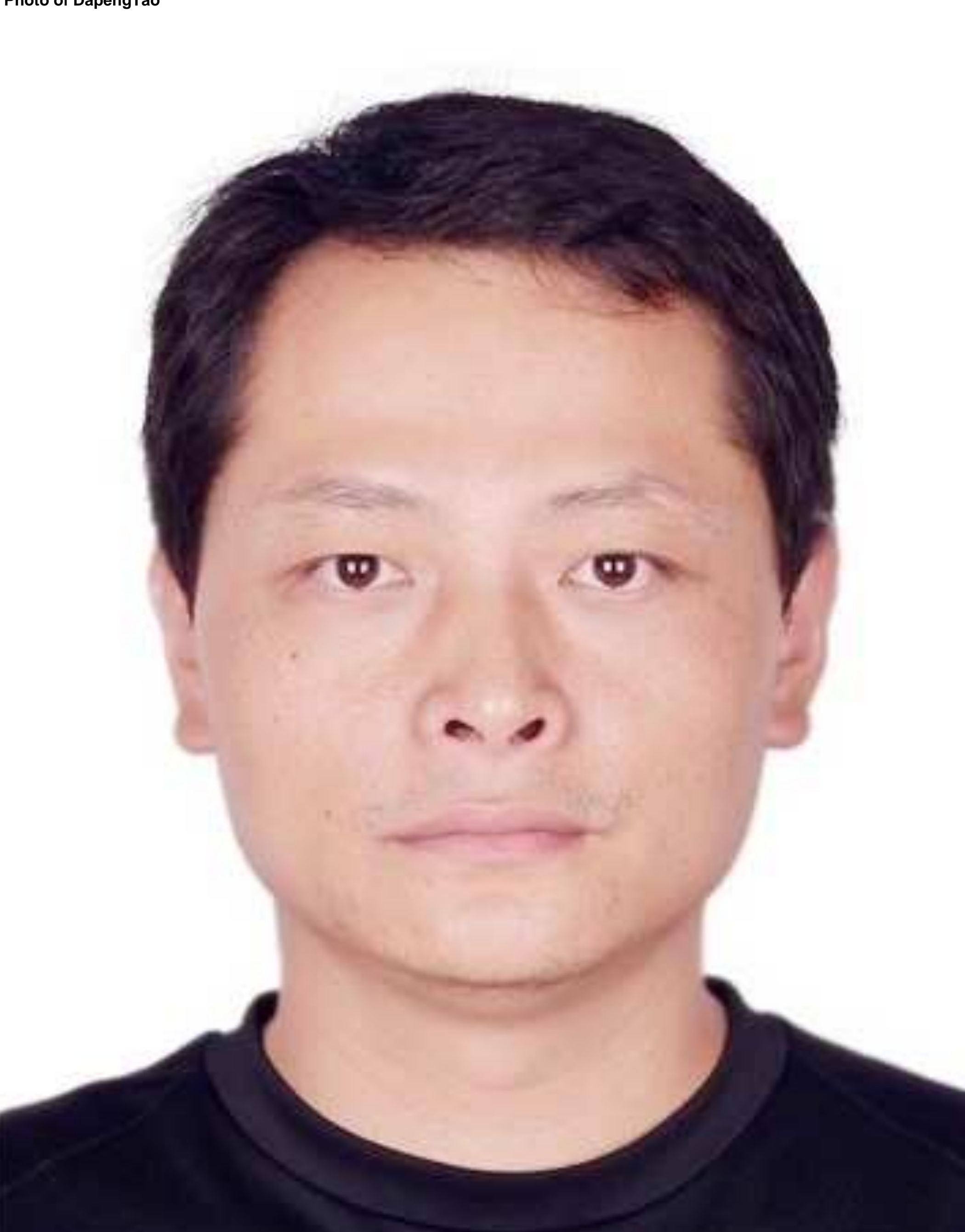